\title{
Management of acute perforated diverticulitis with generalized peritonitis: is this the end of the Hartmann's era?
}

\author{
G. A. Binda ${ }^{1}$
}

Received: 30 March 2020 / Accepted: 30 March 2020 / Published online: 10 April 2020

(c) Springer Nature Switzerland AG 2020

Recent epidemiological studies report a significantly increasing trend in overall hospital admissions for diverticulitis [1] and admissions for complicated diverticulitis [2], especially in younger patients, the most frequent (35\%) complication being peritonitis. These data, together with the decreasing role of surgery in diverticular disease [3], underline the growing need to optimize the surgical strategy to improve the outcomes and avoid permanent stomas, especially in diffuse peritonitis which is the main indication for surgery.

In this issue of Techniques in Coloproctology, Ryan et al. [4] presented a meta-analysis, comparing primary resection and anastomosis (PRA) with Hartmann's procedure (HP) and confirmed the results of similar studies, showing that PRA is associated with less morbidity and leads to a reduced permanent stoma rate [5]. The authors do not limit their analysis to the four prospective randomized trials (PRT) on the subject but include both prospective and retrospective studies, so that we have a complete review of the topic. This new meta-analysis points out some very important limitations of our knowledge about surgical treatment for complicated diverticulitis like the lack of quality of life outcomes, cost effectiveness analysis and, more importantly, the risk of patient and surgeon selection bias, which make a PRT very difficult in the emergency setting.

Undoubtedly, PRTs are considered the gold standard to evaluate the effect of interventions in medicine and are the main basis of a meta-analysis; at the same time, the value of a meta-analysis depends on the quality of the included trials. In this case, the results of the PRTs and, as a consequence, the conclusions of the meta-analysis are questionable from several methodological standpoints. All of the included PRTs were terminated prematurely, mainly due to accrual difficulty, patients submitted to HP had higher mean American Society of Anesthesiologists (ASA) class, Hinchey and

G. A. Binda

gianbinda1@gmail.com

1 BioMedical Institute, Genoa, Italy
Mannheim peritonitis index (MPI) scores, suggesting some selection bias and lack of blinding, and not enough data about surgeons' expertise are reported. The difficulty in performing a PRT in an emergency setting, where the patient's information and consent could be difficult to obtain and the surgeon's expertise is not always adequate, has already been emphasized [6].

Further issues should also be considered: the need for a more precise classification of the patient's performance status and disease stage to guide the surgical decision-making process, the role of protective ileostomy in PRA, the laparoscopic approach [with a possible role for laparoscopic peritoneal lavage (LPL)] and the surgeon's experience in colorectal surgery.

A more precise classification of the patient's status and the stage of disease is required to improve patient selection and clarify the indication for each surgical procedure. In cases of diffuse peritonitis, for which the Hinchey grade has been the prevalent, if not the only, classification tool used in many studies, the first step taken to determine what surgical approach is best is an early preoperative assessment of the risk of septic shock, by identification of hemodynamic instability, physiological status and organ dysfunction, followed by other predictors of poor outcome, such as severe comorbidities, immune deficits, and advanced age. The next step is the intraoperative evaluation of the perforation and peritonitis, i.e., the duration of perforation, the nature of the peritoneal fluid (clear, purulent or fecal), both included in the MPI score, the presence of a visible open perforation, and the anatomical consequences of the period of inflammation prior to surgery, causing fibrosis and matted adhesions which may require careful dissection which may make a colonic resection more difficult and at higher risk of complications as well as edema of the bowel wall and inflammation at the site of the anastomosis which may predispose the anastomosis to leakage and dehiscence [6]. All these factors are key in choosing between laparoscopic lavage, 
PRA with or without diverting stoma or HP via either an open or laparoscopic approach.

What is the role of a diverting ileostomy in PRA patients? While setting up the methodology of our PRT study at the beginning of 2000, we [7] decided to perform it routinely in patients randomized to PRA, to encourage surgeon participation and avoid low accrual: at that time, HP was the most widely used procedure in patients with diffuse peritonitis due to perforated acute diverticulitis. Surgeons still prefer to perform HP in this category of patients: indeed, in the American College of Surgeons NSQIP report, in 2019, only $7.6 \%$ of emergency procedures for acute diverticulitis were PRA [8]. A diverting stoma in patients with PRA, which was always performed in the first three PRTs, is still favored in the latest PRT, the Ladies trial, which started in 2010, in which it was performed at the surgeon's discretion in $73 \%$ of PRA [9]. A covering ileostomy in PRA does not reduce the anastomotic leak rate, but could reduce its otherwise catastrophic effects such as fecal peritonitis and septicemia. An ileostomy is associated with a certain degree of morbidity and also adds to the cost of the entire operation. It should be performed selectively in patients with poorly prepared bowels, coupled with a distal limb washout [10].

Theoretically, to optimize the preventive role of the diverting ileostomy in a patient with severe fecal loading, it would be necessary to perform an intraoperative colonic wash-out, procedure which is difficult and time-consuming in an emergency setting. The four PRTs comparing PRA to HP do not consider this operative step in their analysis; in two [11, 12], colonic lavage is mentioned in the methods section and its execution left to the surgeon's discretion but no data regarding it are reported. Despite some good results in patients with perforated diverticulitis [13], there are only a few comparative studies, with conflicting results, about intraoperative colonic lavage compared to either no bowel preparation or distal limb washout (with or without proximal limb cleansing) performed in the emergency setting, resulting in variable use of colonic lavage and the almost complete absence of recommendation of the procedure.

An initial laparoscopic approach appears to be good option as an intraoperative evaluation of the intraperitoneal stage of disease to determine the surgical strategy, a simple peritoneal lavage in mild peritonitis without evidence of perforation, an easy procedure that can be performed also by surgeon without extensive experience, or a colonic resection feasible also laparoscopically in severe cases but needing major surgical skills [14].

In the early 2000 s, the promising results of LPL in purulent peritonitis [15] convinced many surgeons of the superiority of this treatment method. A proof of this is that in one of the PRTs comparing PRA to HP [12], some investigators, willing to perform LPL, declined trial participation or were no longer enrolling patients with Hinchey stage III peritonitis because they considered LPL a superior approach, making the recruitment process more difficult and leading to a premature termination of the study. Unfortunately, the promising results of LPL reported in early cohort studies were not confirmed in subsequent PRTs. A recent retrospective, multicenter, international study [16] tried to provide a real-life picture, collecting very detailed data on a great number of patients treated in different medical settings and investigating the possible causes of failure of the technique, defined as need for further surgery, which occurred in $25.5 \%$ of patients. A visible overt perforation, significantly related to extensive adhesiolysis, a high ASA class or a high MPI score appeared to be the possible reasons for failure. These findings can be the basis for future prospective, multicenter trials involving a large number of patients in different clinical settings.

On the other hand, an emergency laparoscopic sigmoid resection appears to be feasible in selected patients with Hinchey stage III and IV diverticulitis, with low morbidity and mortality rates and acceptable conversion and reoperation rates, whether using HP or primary anastomosis, also without diversion [17], provided the operation is performed by an experienced laparoscopic surgeon $[18,19]$. Other potential benefits of the laparoscopic approach could be preventing the risk of incisional hernia and reducing the number of postoperative adhesions, thus increasing the chance of successful laparoscopic stoma closure after HP which translated into a higher rate of stoma reversal in one of the studies [18]

Clearly, one variable that should always be considered in studies comparing surgical procedures is the surgeon. The relevance of a surgeon's experience in colorectal surgery was reported in some studies [20-22]: colorectal surgeons are significantly more prone to perform PRA as emergency procedure for acute diverticulitis with peritonitis, with low morbidity and mortality [20, 22].

We have to be realistic and ask ourselves, as suggested by Ryan et al. [23], whether instead of investing resources in small and underpowered randomized controlled trials in some difficult settings like emergency surgery, we should invest them in a large multicenter, prospective, cohort trial, adequately powered, with strict and precise inclusion criteria and a precise decision-making algorithm, which could be a more efficient and realistic way to compare different treatments.

\section{Compliance with ethical standards}

Conflict of interest The author declares that they he has no conflict of interest. 
Ethical approval This article does not contain any studies with human participants or animals performed by any of the authors.

Informed consent For this type of study formal consent is not required.

\section{References}

1. Binda GA, Mataloni F, Bruzzone M, Carabotti M, Cirocchi R, Nascimbeni R, Gambassi G, Amato A, Vettoretto N, Pinnarelli L, Cuomo R (2018) Annibale B Trends in hospital admission for acute diverticulitis in Italy from 2008 to 2015 . Tech Coloproctol 22(8):597-604

2. Amato A, Mataloni F, Bruzzone M, Carabotti M, Cirocchi R, Nascimbeni R, Gambassi G, Vettoretto NP, Pinnarelli L, Cuomo R, Annibale B, Fontana V, Binda GA (2020) Hospital admission for complicated diverticulitis is increasing in Italy, especially in younger patients: a national database study. Tech Coloproctol. 24(3):237-245

3. Biondo $\mathrm{S}$ (2019) The diminishing role of surgery for acute diverticulitis. Br J Surg 106(4):308-309

4. Ryan OK, Ryan ÉJ, Creavin B et al (2020) Systematic review and meta-analysis comparing primary resection and anastomosis versus Hartmann's procedure for the management of acute perforated diverticulitis with generalised peritonitis. Tech Coloproctol. https ://doi.org/10.1007/s10151-020-02172-2

5. Cirocchi R, Afshar S, Shaban F, Nascimbeni R, Vettoretto N, Di Saverio S, Randolph J, Zago M, Chiarugi M, Binda GA (2018) Perforated sigmoid diverticulitis: Hartmann's procedure or resection with primary anastomosis-a systematic review and meta-analysis of randomised control trials. Tech Coloproctol 22(10):743-753

6. Abbas S (2007) Resection and primary anastomosis in acute complicated diverticulitis, a systematic review of the literature. Int $\mathbf{J}$ Colorectal Dis 22:351-357

7. Binda GA, Karas JR, Serventi A, Sokmen S, Amato A, Hydo L, Bergamaschi R, Study Group on Diverticulitis (2012) Primary anastomosis vs nonrestorative resection for perforated diverticulitis with peritonitis: a prematurely terminated randomized controlled trial. Colorectal Dis 14(11):1403-1410

8. Lee JM, Bai P, Chang J, El Hechi M, Kongkaewpaisan N, Bonde A, Mendoza AE, Saillant NN, Fagenholz PJ, Velmahos G, Kaafarani HM (2019) Hartmann's procedure vs primary anastomosis with diverting loop ileostomy for acute diverticulitis: nationwide analysis of 2,729 emergency surgery patients. J Am Coll Surg 229(1):48-55

9. Lambrichts DPV, Vennix S, Musters GD et al (2019) Hartmann's procedure versus sigmoidectomy with primary anastomosis for perforated diverticulitis with purulent or faecal peritonitis (LADIES): a multicentre, parallel-group, randomised, open-label, superiority trial. Lancet Gastroenterol Hepatol 4(8):599-610

10. Wong NY, Eu KW (2005) A defunctioning ileostomy does not prevent clinical anastomotic leak after a low anterior resection: a prospective, comparative study. Dis Colon Rectum 48(11):2076-2079

11. Oberkofler CE, Rickenbacher A, Raptis DA et al (2012) A multicenter randomized clinical trial of primary anastomosis or
Hartmann's procedure for perforated left colonic diverticulitis with purulent or fecal peritonitis. Ann Surg 256(5):819-826 (discussion 826-817)

12. Bridoux V, Regimbeau JM, Ouaissi M et al (2017) Hartmann's procedure or primary anastomosis for generalized peritonitis due to perforated diverticulitis: a prospective multicenter randomized trial (DIVERTI). J Am Coll Surg 225(6):798-8053

13. Regenet N, Pessaux P, Hennekinne S, Lermite E, Tuech JJ, Brehant O, Arnaud JP (2003) Primary anastomosis after intraoperative colonic lavage vs. Hartmann's procedure in generalized peritonitis complicating diverticular disease of the colon. Int $\mathbf{J}$ Colorectal Dis 18:503-507

14. Cirocchi R, Di Saverio S, Weber DG, Taboła R, Abraha I, Randolph J, Arezzo A, Binda GA (2017) Laparoscopic lavage versus surgical resection for acute diverticulitis with generalised peritonitis: a systematic review and meta-analysis. Tech Coloproctol 21(2):93-110

15. Myers E, Hurley M, O'Sullivan GC, Kavanagh D, Wilson I, Winter DC (2008) Laparoscopic peritoneal lavage for generalized peritonitis due to perforated diverticulitis. Br J Surg 95(1):97-101

16. Binda GA, Bonino MA, Siri G, Di Saverio S, Rossi G, Nascimbeni R, Sorrentino M, Arezzo A, Vettoretto N, Cirocchi R, LLO Study Group (2018) Multicentre international trial of laparoscopic lavage for Hinchey III acute diverticulitis (LLO Study). Br J Surg 105(13):1835-1843

17. Dreifuss NH, Schlottmann F, Piatti JM, Bun ME, Rotholtz NA (2020) Safety and feasibility of laparoscopic sigmoid resection without diversion in perforated diverticulitis. Surg Endosc 34(3):1336-1342

18. Vennix S, Lips DJ, Di Saverio S et al (2016) Acute laparoscopic and open sigmoidectomy for perforated diverticulitis: a propensity score-matched cohort. Surg Endosc 30(09):3889-3896

19. Cirocchi R, Fearnhead N, Vettoretto N, Cassini D, Popivanov G, Brandon Michael Henry F, Tomaszewski K, D'Andrea V, Davies J, Di Saverio S (2019) The role of emergency laparoscopic colectomy for complicated sigmoid diverticulits: a systematic review and meta-analysis. Surgeon 17(6):360-369

20. Jafferji MS, Hyman N (2014) Surgeon, not disease severity, often determines the operation for acute complicated diverticulitis. J Am Coll Surg 218(6):1156-1161

21. de Korte N, Klarenbeek BR, Kuyvenhoven JP, Roumen RM, Cuesta MA, Stockmann HB (2011) Management of diverticulitis: results of a survey among gastroenterologists and surgeons. Colorectal Dis 13(12):e411-e417

22. Baldock TE, Brown LR, McLean RC (2019) Perforated diverticulitis in the North of England: trends in patient outcomes, management approach and the influence of subspecialisation. Ann R Coll Surg Engl 101(8):563-570

23. Doleman B, Williams JP, Lund J (2019) Why most published meta-analysis findings are false. Tech Coloproctol 23(9):925-928

Publisher's Note Springer Nature remains neutral with regard to jurisdictional claims in published maps and institutional affiliations. 